\title{
ANÁLISE DA QUALIDADE DE VIDA DOS PACIENTES COM CÂNCER DE LARINGE EM HOSPITAL DE REFERÊNCIA NA REGIÃO SUDESTE DO BRASIL
}

\author{
Quality of life analysis in laryngeal cancer patients \\ at a referral hospital in Southeastern Brazil
}

\author{
Cristina Tostes Vieira Maciel (1), Isabel Cristina Gonçalves Leite (2), \\ Romário Coelho Soares ${ }^{(3)}$, Renata Jacob Daniel Salomão Campos ${ }^{(4)}$
}

\begin{abstract}
RESUMO
Objetivo: estimar a qualidade de vida e fatores a ela associados de uma amostra de pacientes portadores de tumor laríngeo, na região Sudeste do Brasil. Método: amostra constituída por 60 pacientes em tratamento para o câncer de laringe em 2 Hospitais do município de Juiz de Fora, estado de Minas Gerais (Brasil), cidade polo regional e referência para tratamento de cânceres de cabeça e pescoço na região sudeste do Brasil. Com a aplicação do questionário Functional Assessment Cancer Therapy- Head\&Neck, foram coletados os dados e submetidos à análises bivariada e multivariada, para atestar a implicação das variáveis na qualidade de vida dos pacientes de instituições de atenção terciária para tratamento de câncer. Resultados: na análise bivariada, foram significantes as variáveis: sexo; anos de escolaridade; análise do tratamento recebido; acompanhamento fonoaudiológico e nutricional. Após a análise multivariada, ainda demonstraram associação independente: análise do tratamento recebido, tratamento fonoaudiológico e nutricional. Conclusões: a qualidade de vida dos pacientes envolvidos no estudo pode ser avaliada como satisfatória, sendo influenciada pelo bem-estar físico e funcional. O questionário Functional Assessment Cancer Therapy- Head\&Neck, específico para cabeça e pescoço, mostrou que além dos fatores socioeconômicos e clínico-funcionais, os sociodemográficos também estão ligados à qualidade de vida dos portadores de câncer de laringe. Foi demonstrada também a importância do tratamento reabilitador fonoaudiológico e nutricional no ganho da qualidade de vida dos pacientes.
\end{abstract}

DESCRITORES: Epidemiologia; Neoplasias Laríngeas; Qualidade de Vida

(1) Fonoaudióloga Clínica do Hospital Monte Sinai Juiz de Fora/MG; Mestre em Saúde Brasileira pela Universidade Federal de Juiz de Fora (UFJF) - MG, Brasil.

(2) Cirurgiã Dentista; Professora Adjunta da Universidade Federal de Juiz de Fora; Doutora em Saúde Pública pela Escola Nacional de Saúde Pública (FIOCRUZ), Rio de Janeiro - RJ, Brasil.

(3) Monitor aluno da disciplina de Saúde Coletiva e Epidemiologia da Faculdade de Odontologia da Universidade Federal de Juiz de Fora (UFJF)- MG, Brasil.

(4) Fonoaudióloga Clínca do Hospital Oncológico Juiz de Fora/ MG; Mestre em Educação pelo Centro de Estudos Superiores e Mestre em Saúde Brasileira pela Universidade Federal de Juiz de Fora (UFJF) - MG, Brasil.

Financiamento: Universidade Federal de juiz de Fora

Conflito de interesses: inexistente

\section{INTRODUÇÃO}

Os tumores laríngeos ocupam posição de destaque entre as neoplasias que mais interferem na qualidade de vida do paciente. No Brasil, país em que as neoplasias ocupam o segundo lugar dentre as doenças com maior morbimortalidade, com 130 mil óbitos anuais, número superado somente pelas doenças cardiovasculares ${ }^{1,2}$, este grupo de tumores representa $25 \%$ dos tumores de cabeça e pescoço e cerca de $2 \%$ de todos os cânceres.

Dentre todos os tipos de câncer, os tumores de laringe constituem a décima primeira neoplasia maligna mais frequente, atingindo especialmente 
indivíduos do sexo masculino entre a sexta e a sétima décadas de vida ${ }^{1}$ e respondendo por $2,8 \%$ dos novos casos de câncer em homens no mundo. Em uma perspectiva geral, o número estimado de novos casos de câncer de laringe crescerá de 10 milhões, em 2000, para 15 milhões em $2020^{3}$.

A avaliação da qualidade de vida do paciente é de suma importância no tratamento dos pacientes com tumores de cabeça e pescoço, em especial no câncer de laringe, pois esses geralmente causam significante comprometimento estético e funcional ${ }^{4}$. Os questionários abordando temas relativos à saúde geral, ao tratamento e aos sintomas da doença podem ser utilizados como um grande pilar de avaliação do tema qualidade de vida ${ }^{5,6}$. Outra importância nesta avaliação por questionários seria dada pela possibilidade de triagem desses pacientes para situações como depressão, alcoolismo e risco de incapacidade para o trabalho, direcionando o doente para um suporte assistencial adequado 4 .

Atualmente, são vários os instrumentos empregados para analisar o modo de vida dos pacientes com câncer de cabeça e pescoço, mas nenhum deles pode ser considerado o gold-standard". Entretanto, dentre os questionários desenvolvidos neste sentido, pode-se citar um dos mais utilizados, o Functional Assessment Cancer Therapy General (FACT-G), com sua variante específica para cabeça e pescoço (FACT-HN), que além dos aspectos gerais tratados pelos outros instrumentos ainda versa sobre o relacionamento médico-paciente.

O presente artigo objetivou estimar a qualidade de vida e fatores a ela associados de uma amostra de pacientes tratados de tumor laríngeo, na região Sudeste do Brasil.

\section{MÉTODO}

\section{Pacientes}

Em um estudo de corte transversal, foram entrevistados 60 pacientes atendidos em Juiz de Fora - MG, cidade polo de atenção à saúde, situada na região Sudeste do Brasil. Os 60 pacientes correspondem à totalidade de casos no período de estudo que se enquadravam nos critério de inclusão e com os quais foi possível fazer contato para a entrevista (havia dados de identificação suficientes). Os critérios de inclusão foram: faixa etária acima de 18 anos; tumor primário de laringe e, no mínimo, 1 ano de sobrevida (diagnóstico estabelecido entre os anos de 2001 a 2007). Os pacientes foram tratados em 02 instituições hospitalares de referência do município. Foram excluídos os que apresentavam déficits neurológicos ou cognitivos e os que se negaram a assinar o Termo de Consentimento Livre e Esclarecido.

Durante as consultas ambulatoriais, os pacientes foram entrevistados, pelo mesmo examinador, sem a presença do médico assistente e em espaço reservado para este fim, dentro da própria instituição. A mediana entre o tratamento curativo do paciente e a realização da entrevista foi de 25 meses.

Foram utilizados 02 questionários na coleta de dados. O primeiro contendo dados exploratórios, reunindo variáveis sociodemográficas, relativas a estilo de vida; à caracterização do tumor e tratamento adotado, conforme descrito na Tabela 1.

Em seguida aplicou-se o questionário Functional Assessment Cancer Therapy (FACT-HN) criado e validado na língua inglesa e submetido à tradução e adaptação estrutural para o português seguindo as diretrizes da Functional Assessment Chonic Illmess Therapy (FACIT) $)^{5-7}$.

A análise do questionário FACT-HN baseia-se em três índices globais: TOI que envolve o bem estar físico, bem estar funcional e preocupações adicionais em câncer de cabeça e pescoço; o FACTG que envolve o bem estar físico, sociofamiliar, emocional e funcional e o FACTHN que envolve o bem estar físico, sociofamiliar, emocional, funcional e preocupações adicionais de câncer de cabeça e pescoço.

O presente estudo foi aprovado pelo Comitê de Ética da instituição local, sob o parecer de número $308 / 2007$.

\section{Análise dos dados}

Os dados foram digitados e analisados no programa SPSS for Windows, versão 15.0 (STATISTICAL PACKAGE FOR SOCIAL SCIENCES INCORPORATION, 2006). Para a estatística descritiva utilizou-se de medidas de tendência central (média, mediana) e dispersão (desvio-padrão) para as variáveis quantitativas, bem como frequências absolutas e relativas para as variáveis qualitativas. Para a comparação dos escores de qualidade de vida do FACT-HN, entre os diferentes estratos das variáveis sociodemográficas, econômicas, clínico-funcionais foi utilizado o teste ANOVA. Após a análise bivariada, foram selecionadas as variáveis preditoras com valores de $\mathrm{p} \leq 0,20$ para serem submetidos à análise multivariada, realizada por meio da regressão linear múltipla utilizando-se a técnica enter. Permaneceram no modelo multivariado final, aquelas cujos valores de $p$ foram $\leq 0,10$. 
Tabela 1 - Dados sociodemográficos da amostra estudada de pacientes com tumor de laringe, Juiz de Fora, Brasil, 2008

\begin{tabular}{|c|c|c|}
\hline Variáveis & Número de pacientes & $\%$ \\
\hline \multicolumn{3}{|l|}{ Sexo } \\
\hline Masculino & 57 & 95,0 \\
\hline Feminino & 3 & 5,0 \\
\hline \multicolumn{3}{|l|}{ Idade } \\
\hline$<60$ anos & 16 & 26,7 \\
\hline$>60$ anos & 44 & 73,3 \\
\hline \multicolumn{3}{|l|}{ Escolaridade } \\
\hline Até 12 anos de estudo & 46 & 76,7 \\
\hline Acima de 12 anos de estudo & 14 & 23,3 \\
\hline \multicolumn{3}{|l|}{ Estado civil } \\
\hline Casados & 39 & 65,0 \\
\hline Solteiros & 21 & 35,0 \\
\hline \multicolumn{3}{|l|}{ Profissão } \\
\hline Braçal & 41 & 68,3 \\
\hline Não braçal & 19 & 31,7 \\
\hline \multicolumn{3}{|l|}{ Assistência saúde } \\
\hline Sus & 45 & 75,0 \\
\hline Não SUS & 15 & 25,0 \\
\hline \multicolumn{3}{|l|}{$T N M(T)$} \\
\hline $\mathrm{T} 1, \mathrm{~T} 2$ & 28 & 46,7 \\
\hline T3, T4 & 24 & 40,0 \\
\hline$x$ & 8 & 13,3 \\
\hline \multicolumn{3}{|l|}{$T N M(N)$} \\
\hline NO & 29 & 48,3 \\
\hline N1, N2, N3 & 14 & 23,3 \\
\hline $\mathrm{X}$ & 17 & 28,4 \\
\hline \multicolumn{3}{|l|}{ Tratamento } \\
\hline Cirurgia & 5 & 8,5 \\
\hline Quimioterapia & 3 & 5,0 \\
\hline Radioterapia & 9 & 15,0 \\
\hline Cirurgia e quimioterapia & 2 & 2,3 \\
\hline Cirurgia e radioterapia & 19 & 32,2 \\
\hline Quimioterapia e radioterapia & 9 & 15,0 \\
\hline Cirurgia, quimioterapia e radioterapia & 13 & 22,0 \\
\hline \multicolumn{3}{|l|}{ Tipo de cirurgia } \\
\hline Laringectomia total & 23 & $38,0 \%$ \\
\hline Laringectomia parcial & 16 & $26,6 \%$ \\
\hline Não cirúrgico & 21 & $35,4 \%$ \\
\hline
\end{tabular}

Fonte: Dados da pesquisa

Legenda 1: T - extensão do tumor primário

$\mathrm{N}$ - metástase em linfonodo regional

$M$ - metástase à distância

$\mathrm{X}$ - tumor não pode ser avaliado

\section{RESULTADOS}

Variáveis associadas à qualidade de vida de portadores de câncer de laringe

A Tabela 2 descreve todas as variáveis selecionadas para a construção do modelo de regressão linear múltipla $(p \leq 0,20)$ para cada escore constituinte do FACT-HN.

As tabelas de 3 a 5 mostram o resultado da regressão linear multivariada, apresentando as variáveis independentes associadas aos desfechos representados pelos domínios do FACT-HN (TOI, FACTG e FACTHN). 
Tabela 2 - Variáveis associadas à qualidade de vida, nos três escores constituintes do fact-hn, média do escore e significância estatística (p-valor)

\begin{tabular}{|c|c|c|c|c|c|c|}
\hline \multirow{2}{*}{ Variáveis } & \multicolumn{2}{|c|}{ TOI } & \multicolumn{2}{|c|}{ FACTG } & \multicolumn{2}{|c|}{ FACTHN } \\
\hline & Média (dp) & $p$ & Média (dp) & $p$ & Média (dp) & $p$ \\
\hline \multicolumn{7}{|l|}{ Sexo } \\
\hline Masculino & $72,2(16,6)$ & 0,09 & $86,0(18,8)$ & 0,20 & $113,7(23,6)$ & 0,14 \\
\hline Feminino & $55,7(8,1)$ & & $71,8(11,9)$ & & $93,0(15,5)$ & \\
\hline \multicolumn{7}{|l|}{ Escolaridade } \\
\hline Até 12 anos & $68,9(17,4)$ & $<0,01$ & $82,9(20,2)$ & 0,08 & $109(25,1)$ & 0,08 \\
\hline Acima de 12 anos & $80,4(10,0)$ & & $93,5(10,8)$ & & $125,1(13,1)$ & \\
\hline \multicolumn{7}{|l|}{ Raça } \\
\hline Branca & $71,6(16,6)$ & 0,06 & & & & \\
\hline Não Branca & $70,5(17,2)$ & & & & & \\
\hline \multicolumn{7}{|l|}{ Estado civil } \\
\hline Casado & $74,3(14,1)$ & 0,06 & $87,8(16,5)$ & 0,16 & & \\
\hline Solteiro & $66,0(19,7)$ & & $80,6(22,0)$ & & & \\
\hline \multicolumn{7}{|l|}{ Tabagismo } \\
\hline Fumava e fuma & & & $83,0(18,9)$ & 0,02 & $110,4(24,3)$ & 0,07 \\
\hline Nunca fumou & & & $98,0(12,7)$ & & $125,6(22,2)$ & \\
\hline \multicolumn{7}{|l|}{ Ocupação } \\
\hline Braçal & & & $85,5(16,7)$ & 0,04 & & \\
\hline Não Braçal & & & $54,8(23,0)$ & & & \\
\hline \multicolumn{7}{|l|}{ Estadiamento $T$} \\
\hline $\mathrm{T} 1 \mathrm{~T} 2$ & $76,6(14,3)$ & $<0,01$ & $90,2(16,5)$ & 0,04 & $119,3(19,8)$ & 0,02 \\
\hline T3 T4 & $64,3(18,4)$ & & $79,2(21,8)$ & & $103,3(27,4)$ & \\
\hline$x$ & $74,0(12,1)$ & & $68,7(11,3)$ & & $118,0(14,7)$ & \\
\hline \multicolumn{7}{|l|}{ Tipo de tratamento } \\
\hline Isolados & $79,2(8,6)$ & 0,09 & & & & \\
\hline Associados & $68,7(17,9)$ & & & & & \\
\hline \multicolumn{7}{|l|}{ Faria o mesmo } \\
\hline tratamento & $74,3(12,6)$ & 0,15 & $81,1(14,1)$ & 0,16 & $117,0(17,4)$ & 0,15 \\
\hline Sim & $44,7(25,2)$ & & $88,3(29,6)$ & & $74,2(37,1)$ & \\
\hline Não & & & & & & \\
\hline \multicolumn{7}{|l|}{ Traqueostomia } \\
\hline Definitiva & $65,5(17,9)$ & 0,02 & $81,1(19,7)$ & 0,14 & $105,6(25,6)$ & 0,05 \\
\hline Não/provisória & $75,5(14,5)$ & & $88,3(17,7)$ & & $117,8(25,6)$ & \\
\hline \multicolumn{7}{|l|}{ Comunicação } \\
\hline Laríngea & $74,8(15,3)$ & 0,04 & & & $116,7(22,1)$ & 0,09 \\
\hline Não Laríngea & $65,7(17,5)$ & & & & $106,2(25,0)$ & \\
\hline \multicolumn{7}{|l|}{ Fonoaudiologia } \\
\hline Não encaminhado & $78,2(9,2)$ & 0,06 & $92,5(11,3)$ & 0,05 & $122,4(13,5)$ & 0,04 \\
\hline Completou & $70,0(18,2)$ & & $85,0(20,7)$ & & $111,5(25,6)$ & \\
\hline Desistiu & $64,9(18,8)$ & & $52,2(20,1)$ & & $102,5(26,5)$ & \\
\hline \multicolumn{7}{|l|}{ Nutrição } \\
\hline Não encaminhado & $74,1(14,3)$ & $<0,001$ & $88,2(16,3)$ & $<0,01$ & $114,6(20,1)$ & $<0,01$ \\
\hline Completou & $67,6(13,6)$ & & $81,8(15,5)$ & & $122,3(20,3)$ & \\
\hline Desistiu & $24,0(1,4)$ & & $33,0(12,7)$ & & $80.8(9,2)$ & \\
\hline \multicolumn{7}{|l|}{ Psicologia } \\
\hline Não encaminhado & & & $86,7(16,8)$ & 0,01 & $114,6(21,1)$ & 0,01 \\
\hline Completou & & & $94,3(9,0)$ & & $122,3(13,2)$ & \\
\hline Desistiu & & & $59,8(31,4)$ & & $80,8(40,2)$ & \\
\hline
\end{tabular}

Fonte: Dados da pesquisa 
Tabela 3 - Variáveis preditoras da qualidade de vida caracterizada pelo escore TOI, segundo regressão múltipla, pacientes com tumor de laringe, Brasil, 2008

\section{TOI}

Índice total final dos domínios físico, funcional e preocupações adicionais do câncer de cabeça e pescoço

\begin{tabular}{lccc}
\hline Variáveis & $\boldsymbol{\rho}$ & IC 95\% & p-valor \\
\hline $\begin{array}{l}\text { Sexo } \\
\quad \text { Masculino }\end{array}$ & 1,0 & Ref. & 0,082 \\
$\quad \begin{array}{l}\text { Feminino } \\
\text { Análise do tratamento recebido } \\
\quad \text { Teria feito o mesmo }\end{array}$ & $-14,120$ & $-30,126-1,885$ & \\
$\quad$ Não teria feito o mesmo & 1,0 & Ref. & 0,000 \\
Escolaridade & $-24,953$ & $-37,286--12,620$ & \\
$\quad$ Até 12 anos de estudo & & Ref. & 0,024 \\
$\quad$ Acima de 12 anos de estudo & 1,0 & $-17,554--1,307$ & \\
\hline
\end{tabular}

Fonte: Dados da pesquisa

Legenda 3: $\boldsymbol{\rho}$ - Coeficiente da regressão linear

IC - Intervalo de confiança

Tabela 4 - Variáveis preditoras da qualidade de vida caracterizada pelo escore FACTG, segundo regressão múltipla, pacientes portadores do tumor de laringe, Brasil - 2008

FACTG

Índice total final dos domínios físico, social-familiar, emocional e funcional

\begin{tabular}{|c|c|c|c|}
\hline Variáveis & $\rho$ & IC $95 \%$ & p-valor \\
\hline \multicolumn{4}{|l|}{ Sexo } \\
\hline Masculino & 1,0 & Ref. & 0,094 \\
\hline Feminino & $-17,149$ & $-37,313-3,015$ & \\
\hline \multicolumn{4}{|l|}{ Análise do tratamento recebido } \\
\hline Teria feito o mesmo & 1,0 & Ref. & 0,004 \\
\hline Não teria feito o mesmo & $-22,211$ & $-36,986--7,436$ & \\
\hline \multicolumn{4}{|l|}{ Fonoaudiologia } \\
\hline Não encaminhou/ desistiu & 1,0 & Ref. & 0,057 \\
\hline Completou & $-6,425$ & $-13,064-0,213$ & \\
\hline \multicolumn{4}{|l|}{ Escolaridade } \\
\hline Até 12 anos de estudo & 1,0 & Ref. & 0,062 \\
\hline Acima de 12 anos de estudo & $-9,074$ & $-18,612-0,464$ & \\
\hline \multicolumn{4}{|l|}{ Tabagismo } \\
\hline Fumava /continua & 1,0 & Ref. & 0,035 \\
\hline Nunca fumou & $-11,496$ & $-22,175--0,817$ & \\
\hline
\end{tabular}

Fonte: Dados da pesquisa

Legenda 4: $\boldsymbol{\rho}$-Coeficiente da regressão linear

IC - Intervalo de confiança 
Tabela 5 - Variáveis preditoras da qualidade de vida caracterizada pelo escore FACTHN segundo regressão linear múltipla, pacientes com tumor de laringe, Brasil - 2008

\begin{tabular}{|c|c|c|c|}
\hline \multicolumn{4}{|c|}{$\begin{array}{c}\text { FACTHN } \\
\text { Índice total final dos domínios físico, social-familiar, emocional, funcional } \\
\text { e preocupações adicionais do câncer de cabeça e pescoço }\end{array}$} \\
\hline Variáveis & $\rho$ & IC 95\% & p.valor \\
\hline \multicolumn{4}{|l|}{ Análise do tratamento recebido } \\
\hline Teria feito o mesmo & 1,0 & Ref. & 0,000 \\
\hline Não teria feito o mesmo & $-33,784$ & $-51,317--16,251$ & \\
\hline \multicolumn{4}{|l|}{ Fonoaudiologia } \\
\hline Não encaminhou/desistiu & 1,0 & Ref. & 0,103 \\
\hline Completou & $-6,934$ & $-15,322-1,455$ & \\
\hline \multicolumn{4}{|l|}{ Nutrição } \\
\hline Não encaminhou/desistiu & 1,0 & Ref. & 0,103 \\
\hline Completou & $-9,646$ & $-21,313-2,021$ & \\
\hline \multicolumn{4}{|l|}{ Escolaridade } \\
\hline Até 12 anos de estudo & 1,0 & Ref. & 0,015 \\
\hline Acima de 12 anos de estudo & $-14,947$ & $-26,837--3,022$ & \\
\hline
\end{tabular}

Fonte: Dados da pesquisa

Legenda 5: $\boldsymbol{\rho}$-Coeficiente da regressão linear

IC - Intervalo de confiança

\section{DISCUSSÃO}

Inúmeros estudos têm tido como escopo analisar a qualidade de vida nos casos de cânceres de cabeça e pescoço e, particularmente, nos tumores de laringe $\mathrm{e}^{5,7,8-12}$.

São vários os questionários destinados à mensuração da grandeza em discussão, $5,9,10,13-15$, permitindo a avaliação da qualidade de vida tanto no pré quanto no pós tratamento, facultando ao pesquisador o dimensionamento da evolução dos escores após um período terapêutico. Na presente pesquisa, optou-se por utilizar o FACT-HN em consonância com diversos autores ${ }^{5,16,17}$. Este instrumento foi escolhido por ser multidimensional, de fácil aplicação, com um tempo médio de entrevista de 10 minutos, além de ter sido validado para a língua portuguesa ${ }^{4}$. Contudo, ressalta-se a eficiência de todos os instrumentos ${ }^{18}$ que foram utilizados nos artigos supracitados.

Optou-se, neste trabalho, por realizar a entrevista com pacientes com sobrevida de no mínimo 1 ano, pois é o período em que há uma estabilidade no ganho da qualidade de vida, o que foi chamado por alguns autores de platô dos escores ${ }^{10,16}$.

Um exame atento do perfil dos pacientes estudados mostra que a ampla maioria desses era do sexo masculino (57/60), indo ao encontro dos relatos de outras investigações ${ }^{11,15,19}$. A idade também foi condizente com o reportado na literatura (44/60), pois o câncer de laringe foi mais frequente em pacientes acima de 60 anos $^{10,11,16,20}$. A maioria dos pacientes era casada (39/60); trabalhadores braçais (41/60); escolaridade até 12 anos (46/60) e a assistência à saúde mais utilizada por eles foi o Sistema Único de Saúde, sistema de saúde público, universal e gratuito (45/60).

Um prognóstico favorável é dependente do estadiamento da doença, com taxas de sobrevida global para 5 anos de $65 \%-70 \%$ para os tumores nos estádios T1 e T2, cujo tratamento se apresenta compatível com a cura ${ }^{10,19}$. Este estudo apresentou maior frequência de pacientes com o tumor nesses dois estádios, $28(46,7 \%)$, o que mostra de certa forma, um potencial viés de sobrevida na análise. Há demonstrações de que o estádio tumoral quando inicial, possibilita ao paciente uma melhor qualidade de vida ${ }^{10,21-23}$ respaldando os achados na presente análise.

Os tumores nos estádios T3 e T4 sofrem frequentes recidivas e muitos deles não apresentam condições para um novo tratamento curativo, levando à discussão sobre a real necessidade de tratar. Desta forma, a opção pelo não tratamento, pode ser uma alternativa ${ }^{24}$.

Pode-se observar que, via de regra, os tumores mais avançados necessitam de tratamento multimodal (cirurgia, radioterapia e quimioterapia), portanto têm maior probabilidade de apresentarem sequelas e determinar uma pior qualidade de vida ao paciente ${ }^{24}$. 
A forma mais utilizada de tratamento foi a cirurgia e radioterapia combinadas, em $32,2 \%$ dos casos $(n=19)$, concordando com os achados de outros pesquisadores,11,19 De modo geral, a radioterapia ou cirurgia são indicadas para tumores classificados como T1 e T2, já os tumores T3 e T4 requerem terapêutica multimodal, geralmente cirurgia associada à radioterapia adjuvante ${ }^{17}$. O presente estudo, apesar de reunir $46,7 \%$ dos pacientes em estádios 1 e 2 , indicou que modalidades terapêuticas isoladas de cirurgia e radioterapia só foram utilizadas em $8,5 \%$ (5/60) e 15,0\% $(9 / 60)$ dos pacientes, respectivamente.

Concordando com estudo nacional prévio, outras modalidades terapêuticas, ditas alternativas, vêm sendo reportadas na literatura ${ }^{5}$, dentre elas, a ajuda religiosa e espiritual. Dos pacientes analisados $98,3 \%(n=59)$ relataram professar alguma crença religiosa e recorrer à espiritualidade para um conforto face à sua situação de saúde, concordando com o que reportou Fúria (2006) ${ }^{5}$.

No atendimento de pacientes com câncer de laringe, é de suma importância uma equipe multiprofissional, porém não são comuns na literatura internacional trabalhos que enfatizam a influência desta na qualidade de vida dos pacientes. Todavia, neste trabalho, foi notado grande número de indicações de encaminhamento para tratamento reabilitador, compondo uma equipe multidisciplinar no tratamento do doente. A especialidade mais referenciada foi a fonoaudiologia com $68,3 \%$ de encaminhamentos (41 casos), mas destes apenas $28,3 \%(n=17)$ completaram o tratamento.

O paciente encaminhado aos serviços de nutrição e fonoaudiologia, e que completou o tratamento, tende a apresentar uma melhor qualidade de vida. Por outro lado, aqueles que desistiram da terapia ou que, mesmo encaminhados, não aderiram à proposta terapêutica, apresentaram os piores índices de qualidade de vida, influenciando tanto na análise bivariada nos três segmentos TOI, FACTG e FACTHN, bem com na multivariada (o tratamento fonoaudiológico para FACTG e FACTHN; e o tratamento nutricional para FACTHN). Alguns fatores podem estar associados à dificuldade de adesão do paciente aos tratamentos reabilitadores em discussão, como expectativa do paciente; depressão; necessidade de acompanhamento familiar e necessidade de controle dos fatores de risco ${ }^{17}$.

Esta pesquisa ainda mostrou que para os segmentos FACTG e FACTHN, o tratamento fonoaudiológico melhorou a percepção da qualidade de vida. Esta reabilitação beneficia na redução do gap entre a fase do tratamento e o retorno do paciente para o estilo de vida diário, conforme reportaram
Chaukar et al. (2008) ${ }^{2}$. Este achado corrobora com o estudo de Furia (2006)

De um modo geral a qualidade de vida foi satisfatória ao observar os escores ajustados nos três domínios do FACT-HN. O maior impacto da qualidade de vida dos pacientes com câncer laríngeo se refere ao bem-estar geral e às questões específicas do câncer de cabeça e pescoço que dizem respeito à qualidade da voz, dificuldade em deglutir e respirar, além do uso de produtos à base de tabaco e bebidas alcoólicas. Isso mostra que o desempenho diário das funções sociais e das relações familiares do paciente estão ligadas à perspectiva clínico-funcional. Em outros achados descritos na literatura especializada, foram encontrados resultados semelhantes ${ }^{5,17}$, e ressalta-se que nestes foi utilizado o mesmo questionário da presente pesquisa.

A análise bivariada mostrou que as variáveis sexo, escolaridade, estadiamento $T$, presença de traqueostomia, se faria o mesmo tratamento, tratamento fonoaudiológico e nutricional, apresentaram significância estatística $(p \leq 0,20)$ para os três segmentos do FACT-HN (TOI, FACTG, FACTHN). A pesquisa de Fúria $(2006)^{5}$ mostrou resultado semelhante para o fator escolaridade.

No presente trabalho, na análise bivariada, o fator tabagismo associou-se ao FACTG e ao FACTHN como determinante de qualidade de vida, e esta significância permaneceu na análise multivariada, associada somente ao FACTG, se alinhando com o resultado de um estudo prévio realizado no leste europeu $^{20}$. O estudo de FURIA $(2006)^{5}$, no entanto, apresentou o consumo de tabaco associado aos três segmentos do FACT-HN.

$\mathrm{Na}$ análise multivariada, para determinação das variáveis associadas à qualidade de vida nos três domínios, permaneceram: análise do tratamento recebido e escolaridade. Estas variáveis parecem exibir maior impacto na qualidade de vida, pois se apresentou significantemente associadas ao TOI, FACTG e FACTHN, o que se alinhou com os achados de Aastad et al. (2006) ${ }^{10,16,23}$ os quais reportaram diferenças de qualidade de vida relativos a tumores de cabeça e pescoço em pacientes submetidos a diferentes tratamentos. No entanto, a questão análise do tratamento recebido (adesão à mesma proposta terapêutica se fosse oferecida opção de escolha ao paciente) foi a mais significante e positivamente associada à melhor qualidade de vida nos três domínios do questionário, mostrando que a adesão ao tipo de tratamento interfere de maneira efetiva na qualidade de vida da vítima do câncer de laringe.

Apesar do potencial viés de sobrevida, que pode ter superestimado a qualidade de vida da 
amostra analisada, este estudo contribui para o referencial teórico que permite a incorporação de estudos sobre qualidade de vida como um dos itens de avaliação para a determinação da conduta terapêutica e reabilitadora de pacientes com câncer de laringe. Os presentes achados produzem subsídios para a elaboração de protocolos para referenciamento destes pacientes nos centros de atendimento visando o atendimento multidisciplinar e troca de informações entre os profissionais envolvidos. O presente instrumento demonstrou ser de fácil aplicação e aceitação por parte dos pacientes. Foram encontradas maiores dificuldades quando necessárias análises das informações contidas em prontuários, ressaltando a necessidade de uma equipe de saúde comprometida com o registro fidedigno de informações.

\section{CONCLUSÃO}

A qualidade de vida dos pacientes envolvidos no estudo pode ser considerada satisfatória, sendo influenciada tanto por fatores sociodemográficos; socioeconômicos e clínico-funcionais, ainda que de forma diferenciada.

As questões análise do tratamento recebido e anos de escolaridade apresentaram significância na análise multivariada para todos os domínios do FACTHN. Ressalta-se ainda a associação do tratamento fonoaudiológico e do nutricional com a qualidade de vida, relacionada ao FACTHN específico para câncer de cabeça e pescoço.

$O$ adequado referenciamento para serviços multidisciplinares de reabilitação pode ser crítico para minimizar efeitos agudos ou tardios do tratamento para estes tumores, maximizando a função destes pacientes.

\begin{abstract}
Purpose: to estimate the quality of life and associated factors in a sample of patients with laryngeal tumor, in Southeastern Brazil. Method: sample of 60 patients treated for laryngeal cancer in two hospitals in the city of Juiz de Fora, Minas Gerais (Brazil), city and regional center of excellence for the treatment of head and neck cancers in southeastern Brazil. With the application of the questionnaire Functional Assessment Cancer Therapy-Head \& Neck, data were collected and submitted to bivariate and multivariate analysis to demonstrate the involvement of variables in the quality of life of institutions of tertiary care for treatment of cancer. Results: in bivariate analysis ( $p \leq 0.20)$ were significant variables: gender, years of education, analysis of treatment received, speech therapy and nutrition. After multivariate analysis also demonstrated an independent association: analysis of treatment received, speech therapy and nutrition. Conclusions: the quality of life of patients in the study can be evaluated as satisfactory, being influenced by the physical well-being and functional. The questionnaire Functional Assessment Cancer Therapy-Head \& Neck, specific for head and neck, showed that economic factors, clinical and functional, demographic are also linked to quality of life of patients with laryngeal cancer. Also demonstrated was the importance of speech therapy and nutritional rehabilitation treatment gain in quality of life of patients.
\end{abstract}

KEYWORDS: Epidemiology; Laryngeal Neoplasms; Quality of Life

\section{REFERÊNCIAS}

1. Brasil. INCA. Câncer de Laringe. Disponível em: $<$ http://www.inca.gov.br/conteudo_view.asp?id= 332>. Acesso em: 22 jul. 2007.

2. World Health Organization. The Impact of Cancer. Disponível em: <http://www.who.int/ncd surveillance/infobase/web/InfobasePolicymaker/ reports/Rep>. Acesso em: 11 out. 2007.

3. Sartor G, Eluf-Neto J, Travier N, Filho VW, Arcuri ASA, Kowalski et al. Riscos ocupacionais para o câncer de laringe: um estudo caso-controle. Cad Saúde Pública.2007;23:1473-81.

4. Vartanian JG, Carvalho AL, Furia CLB, Castro Júnior $\mathrm{G}$, Rocha $\mathrm{CN}$, Sinitcovisky $\mathrm{IML}$ et al. Questionários para a avaliação da qualidade de vida em pacientes com câncer de cabeça e pescoço validados no Brasil. Rev Bras Cir Cabeça Pescoço. 2007;36(2):108-15.

5. Furia CLB. Qualidade de vida em pacientes tratados de câncer de cavidade oral, faringe e laringe 
em São Paulo: estudo multicêntrico. [doutorado] São Paulo (SP): Universidade de São Paulo; 2006. 6. Saito K, Shiotani A. Laryngeal function after supracricoid laryngectomy. Otolaryngol Head and Neck Surg. 2009;140(4):487-92.

7. Schindler A, Fávero E, Nudo S, Albera R, Schindler $\mathrm{O}$, Cavalo AL. Long-term voice and swallowing modifications after supracricoid laryngectomy: objective, subjective, and self-assessment data. Am J Otolaryngol and Medicine and Surg. 2006;27(6):378-83.

8. Dornfeld K, Simmons JR, Karnell L. Radiation doses to structures within and adjacent to the larynx are correlated with long-term diet-and speechrelated quality of life. Int $\mathrm{J}$ radiation Oncology Biol Phys. 2007;68(3):750-7.

9. Mowry SE, LoTempio MM, Sadeghi A, Wank KH, Wank MB. Quality of life outcomes inlaryngeal and oropharyngeal câncer patients after chemoradiation. Otorinolaryngol. Head and Neck Surg. 2008;135:565-70.

10. Aarstad HJ, Aarstad AKH, Lybak S. The amount of treatment versus quality of life in patients formerly treated for head and neck squamous cell carcinoms. Eur Arch Othorhinolayngol. 2006;263(1):9-25.

11. Van Der Schoeff MP, Derks, W, Hordjk GJH, de Leeuw RJ. The effect of age on survival and quality of life in eldery head and neck cancer patients: a long-term prospective study. Eur Arch Otorhinolaryngol. 2007;264(4):415-22.

12. Donatelli-Lassing AA, Duffy SA, Fowler KE, Ronis DL, Chepeha DB, Terrell JL. The effect of neck dissection on quality of life after chemoradiation. Otolaryngol Head and neck Surg. 2008;139(4):511-8.

13. Thomas L, Jones TM, Tandon $S$ et al. An evolution of the University of Washington Quality of life swallowing domain following oropharyngeal cancer. Eur Arch Otorhinolaryngol. 2008;265(suppl 1):S29-S37.

14. Swinak A, Van Der Brink JL, Wieringa $\mathrm{MH}$ et al. Surgery for recurrent laryngeal carcinoma after radiotherapy: partial laryngectomy or total laryngectomy for a better quality of life? . Otolaryngol Head and Neck Surg. 2005;132(1):95-8.

Recebido em: 05/05/2011

Aceito em: 13/07/2013

Endereço para correspondência:

Isabel Cristina Gonçalves Leite

Rua Silva Jardim no 227/202, Centro

Juiz de Fora - MG

CEP: 36015-390

E-mail: icgleite@ @otmail.com
15. Lotempio MM, Wang KH, Sadeghi A, Delacure $M D$, Juillard DF, Wang MB. Comparison of quality of life outcomes in laryngeal cancer patients following chemoradiation vs. total laryngectomy. Otolaryngol Head and Neck Surg. 2005; 132(6):948-53.

16. List MA, D'Antonio LL, Cella DF, Siston A, Mumby $P$, Haraf $D$ et al. The performance status scale for head and neck cancer patients and the functional assessment of cancer therapy-head and neck scale- a study of utility and validity. Cancer. 1996;77(11):2294-301.

17. Trivedi N, Swaminathan DK, Kuriakose MA et al. Comparison of quality of life in advanced laryngeal cancer patients after concurrent chemotherapy vs total laryngestomy. Otolaryngol Head and neck Surg. 2008;139:702-7.

18. Tschiesner U, Rogers SN, Harreus U, Berghaus A, Cieza A. Content comparison of quality of life quaetionaires used in head and neck cancer based on the international classification of functioning, disability and health: a systematic review. Eur Arch Otorhinolaryngol. 2008;265(6):627-37.

19. Manfro G, Dias FL, Soares JRN, Lima RA, Reis TI. Relação entre idade, sexo, tratamento realizado e estágio da doença com a sobrevida em pacientes terminais com carcinoma epidermoide de laringe. Rev Bras Cancerol. 2006;52(1):17-24.

20. Hashibe M, Boffeta P, Zaridze D, Shangina O, Szeszenia-Dabrowska N, Mates D et al. Contribution of tobacco and alcohol to the high rates of squamous cell carcinoma of the supraglottis in central Europe. Am J Epidemiol. 2007;165(7):814-20.

21. Lazarus CL. Effects of chemoradiotherapy on voice and swallowing. Curr Opin Otolaryngol Head Neck Surg. 2009;17(3):172-8.

22. Chaukar DA, Walvekar RR, Das AK, Deshpande MS, Pai PS, Chaturvedi P et al. Quality of life in head and neck cancer survivors: a cross-sectional survey. Am. J. Otolaryngol. Head and Neck Medicine and Surg. 2009;30(3):176-80.

23. Oozner NB, Benbow J, Downs C, Kelly C, Welch A, Paleri V. The effect of comorbidity on quality of life during radiotherapy in head and neck cancer. Otolaryngol Head and Neck Surg. 2008;139(2):268-72.

24. Tschiesner $U$, Linseinsen $E$, Coenen $M$, Rogers S, Harreus U, Berghaus A et al. Evaluating sequelae after head and neck cancer from the patient perspective with the help of the International Classification of Functioning, Disability and health. Eur Arch Otorhinolaryngol. 2009;266(3):425-36. 